\title{
Rhodnius prolixus en Nicaragua: distribución geográfica, control y vigilancia entre 1998 y 2009
}

\author{
Kota Yoshioka, ${ }^{1}$ Doribell Tercero, ${ }^{1}$ Byron Pérez ${ }^{1}$ y Emperatriz Lugo ${ }^{2}$
}

Forma de citar Yoshioka K, Tercero D, Pérez B, Lugo E. Rhodnius prolixus en Nicaragua: distribución geográfica, control y vigilancia entre 1998 y 2009. Rev Panam Salud Publica. 2011;30(5):439-44.

RESUMEN Objetivo. Presentar la perspectiva general del control de Rhodnius prolixus, el principal vector en la transmisión de la enfermedad de Chagas en Centroamérica, durante el período 1998-2009 en Nicaragua. Describir el control vectorial realizado y presentar la distribución geográfica de las localidades infestadas.

Métodos. Se estudió y analizó la información disponible en el Ministerio de Salud de Nicaragua. Se visualizó la distribución geográfica de R. prolixus mediante el programa visualizador de mapas Quantum GIS 1.5.0.

Resultados. Se determinó que 59 localidades en 14 municipios de 8 departamentos presentaron antecedentes de infestación con R. prolixus entre 1998 y 2009. La altitud de las localidades infestadas oscila entre 160 y 1414 metros sobre el nivel del mar. Se han tratado con más de dos ciclos de control químico 56 localidades. En el segundo ciclo de rociamiento se detectó la presencia del vector, pero durante el tercer ciclo no se lo encontró en ninguna localidad.

Conclusiones. La mayor concentración geográfica de R. prolixus se observó en dos departamentos de la región norte: Madriz y Nueva Segovia. La cantidad de localidades infestadas es menor en Nicaragua que en otros países centroamericanos. El control químico ha sido exitoso en las localidades intervenidas, pero es necesario fortalecer el sistema de vigilancia institucional y comunitaria tanto para vigilar la reinfestación con $\mathrm{R}$. prolixus como para ampliar la cobertura del control vectorial.

Palabras clave Enfermedad de Chagas; Rhodnius; localización geográfica de riesgo; Trypanosoma cruzi; control de vectores; Nicaragua.

En Centroamérica, Rhodnius prolixus Stal (Hemiptera, Reduviidae) es el principal vector de la transmisión al ser humano de Trypanosoma cruzi, agente causal de la enfermedad de Chagas. Se considera que este insecto proviene originalmente de Venezuela y que fue introducido en Centroamérica antes de $1915(1,2)$. Su capacidad vectorial es su-

\footnotetext{
1 Proyecto Chagas, Agencia de Cooperación Internacional del Japón (JICA), Managua, Nicaragua. La correspondencia se debe enviar a Kota Yoshioka,yoshiokakota@gmail.com

2 Dirección de Entomología Médica, Centro Nacional de Diagnóstico y Referencia, Ministerio de Salud de Nicaragua, Managua, Nicaragua.
}

perior a la de Triatoma dimidiata, el vector autóctono de la enfermedad de Chagas en Centroamérica $(3,4)$. El estudio entomológico revela que $R$. prolixus puede eliminarse de las viviendas mediante el control químico (5). Debido a que en Centroamérica la infestación es estrictamente domiciliaria (6-8), la eliminación en gran escala es operativamente factible a través del rociamiento domiciliario con insecticidas de acción residual $(9,10)$. Por tanto, la eliminación del insecto es el objetivo principal para interrumpir la transmisión vectorial de la enfermedad de Chagas en Centroamérica.
El primer informe de la presencia de R. prolixus en Centroamérica fue el de Neiva (11) en El Salvador en 1915. En Nicaragua, según el Ministerio de Salud (MINSA) (12), Arce-Paíz comprobó la presencia del vector en el departamento de Estelí en 1954. Urroz encontró R. prolixus en los departamentos de Matagalpa, Jinotega y Madriz en $1975 .^{3}$

\footnotetext{
3 Citado en: MINSA. Informe emitido del Programa de Control del Mal de Chagas a los departamentos elaborado por Acevedo F., Dirección de Enfermedades Transmitidas por vectores. Managua. Ministerio de Salud de Nicaragua. Diciembre 2001. P.6.
} 
En 1997, los países centroamericanos junto con la Organización Panamericana de la Salud (OPS) lanzaron la Iniciativa de los Países de Centroamérica (IPCA) para la interrupción de la transmisión vectorial y transfusional de T. cruzi (13, 14). La IPCA estableció tres metas para el control de la enfermedad de Chagas: 1) interrupción de la transmisión vectorial por $R$. prolixus, 2) disminución de la transmisión vectorial por $T$. dimidiata y 3) eliminación de la transmisión transfusional de T. cruzi (15).

La IPCA ha definido la eliminación de $R$. prolixus como "la no detección de este vector en un período mínimo de tres años consecutivos, por los métodos disponibles de búsqueda entomológica en áreas con vigilancia instalada y en funcionamiento" (16). Los requisitos establecidos para alcanzar la eliminación de $R$. prolixus son los siguientes: 1) identificar todas las localidades infestadas, 2) rociar el 100\% de las viviendas en todas las localidades infestadas, 3) implementar un programa de información, educación y capacitación en las localidades en áreas de riesgo, 4) implementar y mantener una vigilancia entomológica activa en el $100 \%$ de las localidades infestadas y 5) instalar y mantener un sistema de vigilancia entomológica con la participación comunitaria (16). Asimismo, se recomienda realizar dos ciclos de rociamiento, ya que existe la posibilidad de supervivencia de vectores en los focos no rociados por error (17).

Los países centroamericanos han alcanzado logros en el control de $R$. prolixus. Guatemala obtuvo la certificación de la interrupción de la transmisión vectorial de la enfermedad de Chagas por R. prolixus en 2008 (18). En El Salvador la eliminación de $R$. prolixus fue certificada en 2010 (19). Nicaragua y Honduras están en proceso de certificación de la interrupción de la transmisión vectorial de la enfermedad de Chagas por $R$. prolixus para el año 2011 (20).

El presente estudio tiene como objetivo mostrar una perspectiva general y actualizada sobre el control de $R$. prolixus en Nicaragua. Se describe el control vectorial en el período 1998-2009 y se presenta la distribución geográfica de todas las localidades infestadas con $R$. prolixus. Se analizan las intervenciones realizadas y se proponen las próximas tareas para lograr su eliminación en el futuro.

\section{MATERIALES Y MÉTODOS}

Se recopiló y sintetizó retrospectivamente la información disponible sobre $R$. prolixus en Nicaragua durante el período 1998-2009. Primero, se construyó el historial del control de $R$. prolixus en Nicaragua con base en los documentos publicados (21-25). Segundo, se identificaron todas las localidades con antecedentes de infestación durante el período 1998-2009 revisando los datos disponibles en el Ministerio de Salud de Nicaragua (MINSA). Así, las localidades infestadas fueron identificadas de acuerdo con los siguientes datos: 1) nombre de la localidad, el municipio y el departamento, 2) información geográfica de las localidades, 3) año de primera detección de $R$. prolixus, 4) modalidad del control vectorial, 5) fechas de rociamiento, 6) presencia de $R$. prolixus capturado durante el rociamiento y 7) fuente de información. En los cuadros 1 a 3 se presentan los datos consolidados. Tercero, se visualizó la distribución geográfica de las localidades infestadas con $R$. prolixus mediante el programa Quantum GIS 1.5.0. Las coordenadas fueron extraídas de la base de datos del MINSA, que contiene tanto las coordenadas obtenidas a través del Sistema de Posicionamiento Global como las registradas en los mapas topográficos en escala 1:50000 publicados por el Instituto Nicaragüense de Estudios Territoriales. Por último, se analizaron los datos del control químico para evaluar los resultados de la intervención.

\section{RESULTADOS}

\section{Control de $R$. prolixus en Nicaragua}

El MINSA estableció el programa de control de la enfermedad de Chagas en 1998, dentro del cual se desarrollaron tres etapas: 1) encuesta entomológica nacional, 1998-1999, 2) campaña de eliminación a través del control químico, 1999-2001, y 3) vigilancia institucional y comunitaria, 2002-2009.

Entre 1998 y 1999 el MINSA realizó la encuesta entomológica de triatominos en 15 de los 17 departamentos del país. Se seleccionó el 5\% de las viviendas existentes mediante muestreo sistemático y se encuestaron 32195 viviendas en 3579 localidades de 129 municipios (21). El método usado en la encuesta entomológica fue la búsqueda manual

CUADRO 1. Número de localidades por municipio con antecedentes de infestación por Rhodnius prolixus, Nicaragua, 1998-2009

\begin{tabular}{|c|c|c|c|c|c|}
\hline Departamento & Municipio & $\begin{array}{l}\text { Número de } \\
\text { localidades } \\
\text { infestadas }\end{array}$ & $\begin{array}{l}\text { Rango de } \\
\text { altitud (m) }\end{array}$ & Fuente & $\begin{array}{l}\text { Referencia } \\
\text { en figura } 1\end{array}$ \\
\hline Carazo & La Paz de Carazo & 3 & $160-280$ & $b, c, d$ & $1-3$ \\
\hline Chinandega & San Pedro del Norte & 1 & 680 & $b, c, d$ & 4 \\
\hline Granada & Diria & 1 & 278 & $b, c, d$ & 5 \\
\hline Jinotega & Jinotega & 1 & 828 & $a, b, d, f$ & 6 \\
\hline \multirow[t]{4}{*}{ Madriz } & San José Cusmapa & 9 & $720-1286$ & $a, b, c, d, e, f$ & $7-15$ \\
\hline & Las Sabanas & 1 & 1414 & c & 16 \\
\hline & Telpaneca & 2 & 798-927 & $a, b, d, e, f$ & 17,18 \\
\hline & Totogalpa & 16 & $645-1080$ & $a, b, c, d, f$ & $19-34$ \\
\hline Masaya & Niquinohomo & 4 & $380-410$ & $a, b, c, d$ & $35-38$ \\
\hline Matagalpa & San Ramon & 3 & $920-955$ & $b, c, e, f$ & $39-41$ \\
\hline \multirow[t]{4}{*}{ Nueva Segovia } & Ciudad Antigua & 6 & $628-823$ & $a, b, c, d, e, f$ & $42-47$ \\
\hline & Jicaro & 4 & $615-860$ & $a, b, d, f$ & $48-51$ \\
\hline & Macuelizo & 5 & $706-1218$ & $a, b, c, d, e, f$ & $52-56$ \\
\hline & Mozonte & 3 & $645-705$ & $a, b, c, d, f$ & $57-59$ \\
\hline Total & & 59 & $160-1414$ & & \\
\hline
\end{tabular}

a Ministerio de Salud (MINSA). Informe emitido del Programa de Control del Mal de Chagas a los departamentos elaborado por Acevedo F., Dirección de Enfermedades Transmitidas por vectores. Managua.

b MINSA. Avances de Nicaragua noviembre 1998-Junio 2007. Programa de Chagas. Presentación de PowerPoint expuesta por Marín F. en la X reunión anual de la Iniciativa de los Países Centroamericanos para la interrupción de transmisión de la enfermedad de Chagas (IPCA), 2007. P. 34

"MINSA. Base de datos "Consolidados Rhodnius prolixus" en el Centro Nacional para Diagnóstico y Referencia, Dirección de Entomología Médica. Actualizado hasta el 7 de junio de 2010.

d MINSA. Informe "Control del rociado - Campaña de eliminación de Rhodnius prolixus" entregado a la Organización Panamericana de la Salud en Nicaragua. Programa de mal de Chagas. Dirección de Enfermedades Transmitidas por Vectores. Managua. 2008.

e MINSA. Organización Panamericana de la Salud, Agencia Española de Cooperación Internacional. Informe final "Plan de Acción Operativo para el control de la Enfermedad de Chagas en tres SILAIS endémicos de Nicaragua". 2008.

f MINSA. Materiales en Programa de Enfermedades Transmitidas por Vectores en los departamentos correspondientes. 
CUADRO 2. Número de localidades en las que se detectó Rhodnius prolixus, por año, y su modalidad del control vectorial por la cual se detectó la presencia del vector, Nicaragua, 1998-2009

\begin{tabular}{lll}
\hline Año & $\begin{array}{c}\text { No. de } \\
\text { localidades detectadas }\end{array}$ & \multicolumn{1}{c}{ Modalidad del control vectorial } \\
\hline 1998 & 3 & Encuesta entomológica (2), Vigilancia comunitaria (1) \\
1999 & 17 & Encuesta entomológica (17) \\
2000 & 31 & Rociamiento (31) \\
2001 & 0 & Vigilancia institucional (1), Vigilancia comunitaria (1) \\
2002 & 2 & \\
2003 & 0 & Control de caso positivo (1) \\
2004 & 1 & Rociamiento complementario (2) \\
2005 & 0 & Vigilancia comunitaria (1) \\
2006 & 0 & \\
2007 & 2 & \\
2008 & 2 & \\
2009 & 1 & \\
Total & 59 & \\
\hline
\end{tabular}

Nota: las cifras en paréntesis indican el número de localidades detectadas a través de la modalidad correspondiente.

\begin{abstract}
CUADRO 3. Número de localidades rociadas y número de localidades que presentaron Rhodnius prolixus al momento del rociamiento, por ciclo de rociamiento, Nicaragua, 1998-2009
\end{abstract}

\begin{tabular}{ccc}
\hline $\begin{array}{c}\text { Ciclo de } \\
\text { rociamiento }\end{array}$ & $\begin{array}{c}\text { No. de } \\
\text { localidades rociadas }\end{array}$ & $\begin{array}{c}\text { No. de localidades } \\
\text { rociadas en las } \\
\text { que se detectó } R \text {. prolixus }\end{array}$ \\
\hline 1 & 59 & 51 \\
2 & 56 & 11 \\
3 & 20 & 0 \\
\hline
\end{tabular}

a Veces que se llevó a cabo el rociamiento de las viviendas.

por hombre-hora (26). Como resultado, se registró la presencia de $R$. prolixus en 20 localidades de 5 departamentos: Chontales, Jinotega, Madriz, Masaya y Nueva Segovia (21). Sin embargo, el espécimen del departamento de Chontales fue identificado posteriormente como Rhodnius pallescens (22). El estudio de Zeledón et al. (2006) también destaca la ausencia de $R$. prolixus en este departamento (23). En total se identificaron 19 localidades infestadas a través de la encuesta entomológica nacional 1998-1999. En la misma encuesta, el índice de infestación (número de viviendas infestadas/ número de viviendas encuestadas $\times 100$ ) (26) con $R$. prolixus en los departamentos de Madriz, Nueva Segovia, Masaya y Jinotega fue $1,0 \%, 0,5 \%, 0,03 \%$ y $0,03 \%$, respectivamente (21).

Entre 1999 y 2001 el MINSA realizó una campaña de eliminación de $R$. prolixus a través de dos ciclos de control químico. El primer ciclo de rociamiento fue realizado en las 20 localidades donde se encontró el vector hasta el año 1999 y en las 68 localidades circundantes, dentro de un radio de $3 \mathrm{~km}$, que presenta- ban características similares. Al realizar el primer ciclo de rociamiento se detectó $R$. prolixus en 31 de las 68 localidades circundantes rociadas. El segundo ciclo se realizó en las 20 localidades detectadas hasta el año 1999 y en las 29 localidades detectadas mediante el primer ciclo de rociamiento ${ }^{4}$ (22). Se consideró como objetivo del control a $100 \%$ de las viviendas en las localidades programadas. El rociamiento fue realizado por el personal profesional del MINSA con una bomba aspersora manual (Hudson X-Pert ${ }^{\circledR}$ de 8 litros). Los insecticidas utilizados y sus dosis de ingrediente activo (i.a.) fueron etofenprox $0,250 \mathrm{~g}$ i.a. $/ \mathrm{m}^{2}$, $\lambda$-cihalotrina $0,030 \mathrm{~g}$ i.a. $/ \mathrm{m}^{2}, \beta$-ciflutrina 0,025 g i.a. $/ \mathrm{m}^{2}$, deltametrina 0,025 g i.a./ $\mathrm{m}^{2}$ y ciflutrina $0,050 \mathrm{~g}$ i.a. $/ \mathrm{m}^{2}$. Se roció del interior de las viviendas y el peri-

\footnotetext{
4 MINSA. Informe "Control del rociado - Campaña de eliminación de Rhodnius prolixus" entregado a la Organización Panamericana de Salud en Nicaragua. Programa de mal de Chagas. Dirección de Enfermedades Transmitidas por Vectores. Managua. 2008. Nota: No se incluye el número de localidades rociadas con el objetivo de controlar T. dimidiata.
}

domicilio, y en promedio se consumieron 16 litros de suspensión (dos cargas de la bomba aspersora) por vivienda. Al cabo de la campaña de eliminación 1999-2001, 49 localidades infestadas en 14 municipios se rociaron dos veces. El número de viviendas rociadas en el primer y el segundo ciclos fue de $3106 \mathrm{y}$ 3104 , con coberturas de $96,6 \%$ y $95,8 \%$, respectivamente. $^{5}$

En el año 2002 comenzó la etapa de vigilancia, que consiste en la vigilancia institucional, la vigilancia comunitaria y el rociamiento complementario. Para la vigilancia institucional, el MINSA realizó esporádicamente encuestas entomológicas y trató de establecer la vigilancia comunitaria para promover el reporte del vector por parte de las comunidades. Sin embargo, el presente estudio no pudo determinar el nivel de funcionamiento del sistema de vigilancia. No obstante, a través de la vigilancia se detectaron 3 nuevas localidades infestadas con $R$. prolixus en los años 2002 y 2004 en los departamentos de Madriz, Matagalpa y Nueva Segovia.

En 2007 y 2008, el MINSA ejecutó un plan de rociamiento complementario en las cuatro localidades en las que había quedado pendiente llevar a cabo dos ciclos de rociamiento, incluyendo 22 localidades circundantes. Al realizar el rociamiento se detectó $R$. prolixus en cuatro localidades más en los departamentos de Madriz y Matagalpa. (24).

Además del control vectorial, el MINSA realizó una encuesta serológica en el año 2003. Las muestras fueron obtenidas de niños menores de 15 años en los municipios infestados. La seroprevalencia a nivel municipal fue de $0,0 \%$ a $19,7 \%$; los valores más altos se encontraron en los departamentos de Madriz y Nueva Segovia $(10,8 \%$ y $4,5 \%$, respectivamente) (25).

\section{Localidades con antecedentes de infestación con $R$. prolixus}

En el cuadro 1 se presenta el número de localidades con antecedentes de infestación durante el período 1998-2009 en Nicaragua. Se ha encontrado $R$. prolixus en un total de 59 localidades en 14 municipios de 8 departamentos. La altitud

\footnotetext{
MINSA. Avances de Nicaragua Nov. 1998 - Junio 2007. Programa de Chagas. Presentación de PowerPoint expuesta por Marín F. en la X reunión anual de la Iniciativa de los Países Centroamericanos para la interrupción de transmisión de la enfermedad de Chagas (IPCA), 2007. Pp. 12-13.
} 
promedio sobre el nivel del mar fue de $801 \mathrm{~m}$, con un rango de 160-1414 m.

En el cuadro 2 se muestra que la detección del vector se concentra en los años 1999 y 2000 en el transcurso de 12 años. Esta concentración temporal se debe a la ejecución del control organizado, es decir, a través de la encuesta entomológica nacional y la campaña de eliminación mediante el control químico masivo. Posteriormente, la detección fue esporádica mediante la vigilancia y el control selectivo. En el año 2009 se encontró el vector en una localidad del departamento de Madriz, y este representa el último registro de $R$. prolixus en Nicaragua hasta el año 2009.

\section{Distribución geográfica de las localidades infestadas con $R$. prolixus}

En la figura 1 se muestra la distribución geográfica de las localidades infestadas. Es evidente que la distribución de las localidades infestadas se extendió progresivamente alrededor de las localidades detectadas en la encuesta entomológica nacional 1998-1999. Hasta el año 2009, se detectó el vector en 59 localidades y la mayor concentración se observó en los departamentos de Madriz y Nueva Segovia. En los otros 7 departamentos infestados la distribución de localidades con $R$. prolixus es limitada.

\section{Control químico en las localidades infestadas con $R$. prolixus}

En 56 de las 59 localidades en las que se detectó $R$. prolixus se completaron más de dos ciclos de rociamiento (cuadro 3) hasta finales de 2009. En el momento del segundo ciclo de rociamiento se detectó el vector en 11 de las 56 localidades rociadas, lo que implica la persistencia de la infestación a pesar del primer rociamiento. En el tercer rociamiento no se capturaron insectos en ninguna de las 20 localidades rociadas. Esta evidencia sustenta la recomendación de realizar dos ciclos de rociamiento para eliminar la infestación de $R$. prolixus (17).

\section{DISCUSIÓN}

En Nicaragua, la distribución geográfica de $R$. prolixus se circunscribe a ciertas localidades en la forma focalizada. Se observaron dos concentraciones geográficas en el sur del departamento de Madriz y a lo largo del límite departamental

FIGURA 1. Distribución geográfica de las localidades en las que se detectó $R$. prolixus. Nicaragua, 1998-2009
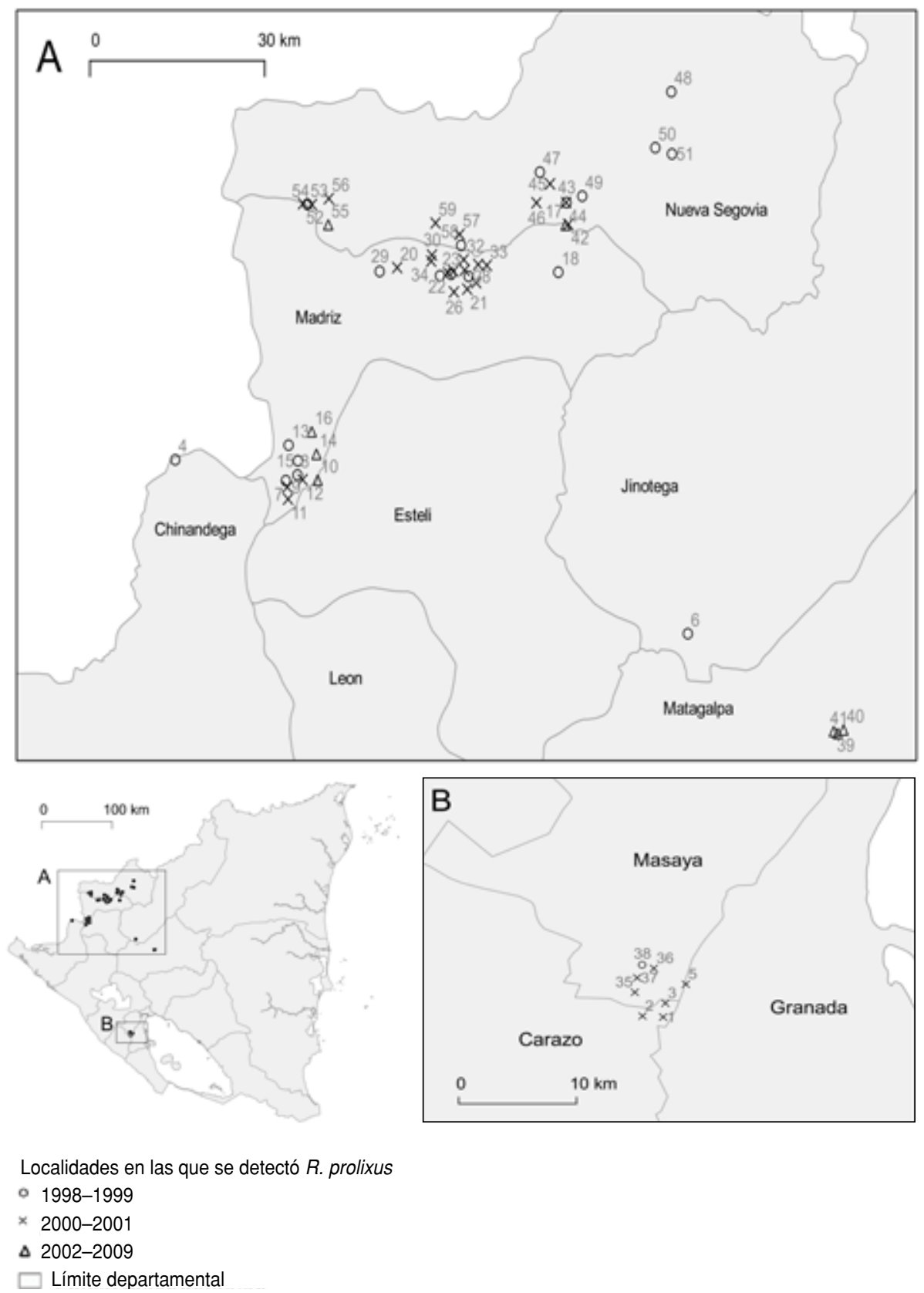

Nota: Los círculos representan localidades en las que se detectó el vector en 1998-1999; las cruces, en 2000-2001; y los triángulos, en 2002-2009. Los números se corresponden con los de la última columna del cuadro 1.

entre Madriz y Nueva Segovia. En otras zonas las localidades infestadas se sitúan como enclaves aislados. Es notable haber identificado localidades infestadas con una altitud de 160-410 m sobre el nivel del mar; en el estudio entomológico nacional llevado a cabo en Guatemala no se detectó el vector por debajo de los $600 \mathrm{~m}$ (27). Posiblemente, la altitud es un indicador tentativo para pronosticar la dis- tribución de $R$. prolixus. Por ejemplo, el análisis geográfico en Guatemala indica que la humedad y la temperatura están asociadas con la presencia de $R$. prolixus más que la altitud (28).

Un hecho que debe destacarse es que la cantidad de localidades con antecedentes de infestación por $R$. prolixus es menor en Nicaragua que en Guatemala y Honduras. En Guatemala se confirmó 
la presencia del vector en 317 localidades durante los años 2000-2007 (18) y en Honduras se identificaron 517 localidades infestadas hasta el año 2010 (29). La razón de esta diferencia puede ser compleja, y se pueden considerar las siguientes hipótesis: 1) la cantidad de localidades en las zonas de riesgo es menor, 2) la migración de $R$. prolixus desde los países cercanos disminuyó, 3) el hábitat de $R$. prolixus fue limitado por las características de las viviendas, y 4) el alcance geográfico del control institucional fue insuficiente para revelar todas las localidades infestadas.

El alcance geográfico del control vectorial no está completamente definido. El hallazgo de $R$. prolixus en nuevas localidades durante los años 2002-2009 implica que el número de localidades intervenidas durante los años 1998-2002, incluyendo la encuesta entomológica y el sucesivo control químico, no fue suficiente para identificar todas las localidades infestadas. Por ejemplo, en el departamento de Matagalpa no se detectó $R$. prolixus en la encuesta entomológica 1998-1999 y, por lo tanto, no se programó el control químico hasta que se encontró una localidad infestada en el año 2002. En este sentido, probablemente existan localidades infestadas en las zonas que han quedado fuera del alcance del control vectorial realizado.

El funcionamiento de la vigilancia es parcial pese a que se registraron tres informes comunitarios de detección de $R$. prolixus durante los años 1998-2009. Esto se debe a que en el MINSA no existe una estrategia concreta y consecuente para mantener el sistema de vigilancia, y por lo tanto las actividades de vigilancia no se han llevado a cabo de manera periódica. Es necesario fortalecer el sistema de vigilancia, tanto para vigilar la reinfestación con $R$. prolixus en las localidades intervenidas como para ampliar la cobertura del control vectorial. Para la vigilancia institucional, es imprescindible continuar con la encuesta entomológica en las localidades consideradas prioritarias según los factores de riesgo de infestación con $R$. prolixus, tales como la seroprevalencia, la tipificación de la vivienda, el índice entomológico, etc. (25). Asimismo, es recomendable mejorar la calidad de la encuesta entomológica mediante la aspersión de un irritante químico (flushing out), ya que es un método más sensible para detectar el vector $(5,30)$. La vigilancia con participación comunitaria también es de suma importancia para sostener el impacto del rociamiento y asegurar la eliminación de la infestación (16). Involucrar a los niños escolares en la búsqueda de los vectores puede ser efectivo para mejorar la calidad de la vigilancia comunitaria (31). En general, para administrar la vigilancia en forma sostenible, es indispensable trazar el diseño del sistema de vigilancia de acuerdo a la tendencia de descentraliza- ción e integración en el sistema de salud en Nicaragua.

Este estudio presentó limitaciones en las fuentes de información y los datos obtenidos. El análisis del control vectorial en las viviendas fue imposible porque se carece de datos que reflejen el número de viviendas existentes, encuestadas, infestadas o rociadas por año o por localidad. Además, los registros existentes presentan datos confusos y muestran incongruencias entre ellos. El alcance de este estudio es presentar una perspectiva general hasta el nivel de las localidades, sin analizar los datos en el nivel de las viviendas.

En conclusión, el control de R. prolixus ha sido exitoso en Nicaragua, por lo menos en las localidades donde se ha detectado la infestación y se han completado dos ciclos de rociamiento. Sin embargo, todavía hay localidades donde no se ha evaluado la situación entomológica y se desconoce si hay infestación de vectores. En estas localidades, no se puede descartar la existencia de infestación con $R$. prolixus. Las futuras tareas deberán fortalecer la vigilancia comunitaria e institucional y ampliar el alcance geográfico del control vectorial.

Agradecimientos. Al Ministerio de Salud de Nicaragua por suministrar datos e información, especialmente a Francisco Acevedo, William Chacón y María Eugenia Parrales.

\section{REFERENCIAS}

1. Dujardin J, Muñoz M, Chavez T, Ponce C, Moreno J, Schofield C. The origin of Rhodnius prolixus in Central America. Medical and Veterinary Entomology. 1998;12:113-5

2. Schofield C. Challenges of Chagas Disease Vector Control in Central America. Position paper. Global collaboration for development of pesticides for public health (GCDPP), WHO/CDS / WHOPES / GCDPP / 2000.1. World Health Organization; 2000.

3. Ponce C, Ponce E, Avila MFG, Bustillo O. Ensayos de intervención con nuevas herramientas para el control de la Enfermedad de Chagas en Honduras. En: Nuevas Estrategias para el Control Vectorial de la Enfermedad de Chagas en Honduras. Tegucigalpa: Ministerio de Salud Pública de Honduras; 1995. Pp. 10-6.

4. Paz-Bailey G, Monroy C, Rodas A, Rosales R, Tabaru Y, Davies C, et al. Incidence of Trypanosoma cruzi infection in two Guatemalan communities. Trans R Soc Trop Med Hyg. 2002;96(1):48-52.

5. Tabaru Y, Monroy C, Rodas A, Mejía M, Rosales R. Chemical control of Triatoma dimidiata and Rhodnius prolixus (Reduviidae: Triatominae), the principal vectors of Chagas' Disease in Guatemala. Med Entomol Zool. 1998;49(2):87-92.

6. Schofield C, Dujardin J. Chagas Disease Vector Control in Central America. Parasitology Today. 1997;13(4):141-4.

7. Monroy C, Rodas A, Mejía M, Rosales R, Tabaru Y. Epidemiology of Chagas Disease in Guatemala: Infection Rate of Triatoma dimidiata, Triatoma nitida and Rhodnius prolixus (Hemiptera, Reduviidae) with Trypanosoma cruzi and Trypanosoma rangeli (Kinetoplastida, Trypanosomatidae). Mem Inst Oswaldo Cruz. 2003;98(3):305-10.

8. Zeledón R. Some historical facts and recent issues related to the presence of Rhodnius prolixus (Stal, 1859) (Hemiptera: Reduviidae) in Central America. Entomol Vect. 2004;11(2): 233-46.

9. Dias J, Silveira A, Schofield C. The Impact of Chagas Disease Control in Latin America - A Review. Mem Inst Oswald Cruz. 2002;97(5):603-12.
10. Nakagawa J, Cordón-Rosales C, Juárez J, Itzep C, Nonami T. Impact of residual spraying on Rhodnius prolixus and Triatoma dimidiata in the Department of Zacapa in Guatemala. Mem Inst Oswaldo Cruz. 2003;98(2):277-81.

11. Neiva A. Contribuição para o conhecimento dos Hemipteros hematofagos da América Central (Nota Previa). Brasil Medico. 1915; 29:1-3. Citado por: Schofield C. Challenges of Chagas Disease Vector Control in Central America. Position paper. Global collaboration for development of pesticides for public health (GCDPP), WHO/CDS/WHOPES/ GCDPP/2000.1. World Health Organization; 2000. P. 9.

12. Nicaragua, Ministerio de Salud (MINSA). Situación epidemiológica de la Enfermedad de Chagas en el SILAIS Madriz, Nicaragua. Boletín epidemiológico Semana 14 Año 2005. Managua: Ministerio de Salud de Nicaragua; 2005.

13. Organización Mundial de la Salud (OMS). Informe Final: Reunión sobre Vectores de la Enfermedad de Chagas en los Países de 
Centroamérica. Tegucigalpa: Organización Mundial de la Salud; 1997.

14. Ponce C. Current situation of Chagas disease in Central America. Mem Inst Oswaldo Cruz, Rio de Janeiro, 2007;102(Suppl.1):41-4

15. Organización Panamericana de la Salud (OPS). Primera Reunión de la Comisión Intergubernamental de la Iniciativa de Centroamérica y Belice para la interrupción de la Transmisión Vectorial de la Enfermedad de Chagas por Rhodnius prolixus, Disminución de la Infestación Domiciliar por Triatoma dimidiata, y Eliminación de la Transmisión Transfusional del Trypanosoma cruzi. Guatemala: Organización Panamericana de la Salud; 1999. (OPS/HCP/HCT/145/99).

16. Organización Panamericana de la Salud (OPS). Informe final: Reunión Internacional para el Establecimiento de Criterios de Certificación de la Eliminación de Rhodnius prolixus. Guatemala: Organización Panamericana de la Salud; 2003. (OPS/DPC/CD/276/03).

17. Nakagawa J. Pauta operativa para la eliminación de Rhodnius prolixus en Centroamérica: Propuesta para la Reunión Internacional para el Establecimiento de Criterios de Certificación de la Eliminación de Rhodnius prolixus. En: OPS. (2003) Informe final: Reunión Internacional para el Establecimiento de Criterios de Certificación de la Eliminación de Rhodnius prolixus. Guatemala: Organización Panamericana de la Salud; 2003. P. 48. (OPS/DPC/ $\mathrm{CD} / 276 / 03)$.

18. Organización Panamericana de la Salud/ Organización Mundial de la Salud. Informe para la Certificación de la Interrupción de la Transmisión Vectorial de la Enfermedad de Chagas por Rhodnius prolixus en la República de Guatemala. Comisión Internacional de Evaluación con Secretaría Técnica, Organización Panamericana de Salud, Organización Mundial de la Salud, Guatemala; 2008.
19. Organización Panamericana de la Salud/ Organización Mundial de la Salud. Informe para la Certificación de la Eliminación del Rhodnius prolixus en El Salvador. El Salvador: Comisión Internacional de Evaluación con Secretaría Técnica, Organización Panamericana de la Salud, Organización Mundial de la Salud; 2010

20. Moncayo A, Silveira A. Current epidemiological trends for Chagas disease in Latin America and future challenges in epidemiology, surveillance and health policy. Mem Inst Oswaldo Cruz. 2009;104(Suppl. I):17-30.

21. Lugo E, Marín F. Resultados de una encuesta entomológica de triatominos (heteróptera: reduviidae: triatominae) realizada en 15 departamentos de Nicaragua, 1998-1999. Rev Nica Ent. 2005;65:1-12.

22. Marín F. Control de Rhodnius prolixus en Nicaragua. En: OPS. (2003) Informe final: Reunión Internacional para el Establecimiento de Criterios de Certificación de la Eliminación de Rhodnius prolixus. Guatemala: Organización Panamericana de la Salud; 2003. P. 17. (OPS/ $\mathrm{DPC} / \mathrm{CD} / 276 / 03)$.

23. Zeledón R, Marín F, Calvo N, Lugo E, Valle S. Distribution and ecological aspects of Rhodnius pallescens in Costa Rica and Nicaragua and their epidemiological implications. Mem Inst Oswaldo Cruz. 2006;101(1):75-9.

24. MINSA/OPS/AECI. Informe final del Plan de acción operativo para el control de la Enfermedad de Chagas en 3 SILAIS endémicos de Nicaragua. Managua: Ministerio de Salud de Nicaragua, Organización Panamericana de la Salud, Agencia Española de Cooperación Internacional; 2008.

25. OPS. Primera Evaluación Internacional del Programa de Control de la Enfermedad de Chagas en Nicaragua, Managua, Matagalpa y Madriz: Organización Panamericana de la Salud; 2003. (OPS/HCP/HCT/03).
26. Schofield C. Field testing and evaluation of insecticides for indoor residual spraying against domestic vectors of Chagas disease. Global collaboration for development of pesticides for public health (GCDPP), WHO/CDS/ WHOPES/GCDPP/2001.1. World Health Organization; 2001.

27. Tabaru Y, Monroy C, Rodas A, Mejía M, Rosales $R$. The geographical distribution of vectors of Chagas' disease and populations at risk of infection in Guatemala. Med Entomol Zool. 1999;50(1):9-17.

28. Bustamante DM, Monroy C, Rodas A, Juarez J, Malone J. Environmental determinants of the distribution of Chagas disease vectors in south-eastern Guatemala. Geospatial Health. 2007;2:199-211.

29. Honduras, Secretaría de Salud. Informe para la Comisión Internacional de Evaluación con la Secretaría Técnica de OPS/OMS para obtener la Certificación de la Interrupción de la Transmisión de la Enfermedad de Chagas por el vector Rhodnius prolixus en Honduras. Managua: Secretaría de Salud; 2010. P. 35.

30. Monroy C, Mejía M, Rodas A, Hashimoto T, Tabaru Y. Assessing methods for the density of Triatoma dimidiata, the principal vector of Chagas' disease in Guatemala. Med Entomol Zool. 1998:49(4):301-7.

31. Hashimoto K, Kojima M, Nakagawa J, Yamagata Y. Effectiveness of Health Education through Primary School Teachers; Activities of Japan Overseas Cooperation Volunteers in the Control of Chagas' Disease Vectors in Guatemala. Technology and development. 2005;18:71-6.

Manuscrito recibido el 13 de enero de 2011. Aceptado para publicación, tras revisión, el 17 de abril de 2011.

ABSTRACT Objective. Present an overview of the control of Rhodnius prolixus, the principal vector in the transmission of Chagas' disease in Central America, during the period 1998-2009 in Nicaragua. Describe the vector control carried out and the geographical distribution of the infested localities.

Rhodnius prolixus in Nicaragua: geographical distribution, control, and surveillance, 1998-2009

Methods. The available information in Nicaragua's Ministry of Health was studied and analyzed. The geographical distribution of $R$. prolixus was visualized using the Quantum GIS 1.5.0 map visualization program.

Results. It was determined that 59 localities in 14 municipalities of 8 departments had a history of $R$. prolixus infestation between 1998 and 2009. The altitude of the infested localities ranges between 160 and 1414 meters above sea level. A total of 56 localities have been treated with more than two cycles of chemical control. The presence of the vector was detected in the second spraying cycle, but it was not found in any locality during the third cycle.

Conclusions. The greatest geographical concentration of $R$. prolixus was found in two departments in the northern region: Madriz and Nueva Segovia. There were fewer infested localities in Nicaragua than in other Central American countries. Chemical control has been successful in the localities treated, but the institutional and community surveillance system needs to be strengthened to monitor $R$. prolixus reinfestation and expand vector control coverage.

Key words Chagas disease; Rhodnius; geographical localization of risk; Trypanosoma cruzi; vector control; Nicaragua. 